\title{
CANCER
}

\section{Tumors secrete gaseous sulfur-containing compounds}

Volatile gaseous sulfur-containing compounds are produced by tumors and stimulate tumour proliferation, new findings show.

Glycolytic metabolism is accelerated in cancer cells, resulting in the accumulation of glucose and methionine in these cells. In this study, the nonenzymatic Maillard reaction between glucose and methionine was found to result in the generation of sulfur-containing compounds, including hydrogen sulfide and methanethiol.

"There has been little research investigating the relationship between the production of volatile substances in tumor tissues and the proliferation and/ or metastasis of cancer," explains Kazue Yamagishi, corresponding author.

Yamagishi et al. compared the levels of sulfur-containing compounds in flatus samples from patients with colon cancer and exhaled air samples from patients with lung cancer with samples from healthy individuals. The results showed that sulfur-containing compounds were found at significantly greater levels in patients with cancer than in healthy individuals.

The researchers also used a mouse model to investigate the effect of these gaseous sulfur-containing compounds on tumor proliferation and metastasis. They found that "tumor proliferation was suppressed by preventing the diffusion of these gases, without using any specific anticancer agent".

"We are planning research that will develop new approaches to anticancer therapy, such as direct desulfurization (of the blood) for rapid detoxification of sulfur-containing gases," says Yamagishi.

Katherine Smith

Original article Yamagishi, K. et al. Generation of gaseous sulfur-containing coumpounds in tumour tissue and suppression of gas diffusion as an antitumour treatment. Gut doi:10.1136/gutjnl-2011-300721 